\title{
Recombinant production of plant lectins in microbial systems for biomedical application - the frutalin case study
}

\section{Carla Oliveira, José A. Teixeira and Lucília Domingues*}

Centre of Biological Engineering, University of Minho, Braga, Portugal

Edited by:

Els Jm Van Damme, Ghent University, Belgium

\section{Reviewed by:}

Wim Van Den Ende, Katholieke Universiteit Leuven, Belgium Alexander D. Frey, Aalto University, Finland

\section{*Correspondence}

Lucília Domingues, Centre of Biological Engineering, University of Minho, Campus de Gualtar,

4710-057 Braga, Portugal

e-mail: luciliad@deb.uminho.pt
Frutalin is a homotetrameric partly glycosylated $\alpha$-D-galactose-binding lectin of biomedical interest from Artocarpus incisa (breadfruit) seeds, belonging to the jacalin-related lectins family. As other plant lectins, frutalin is a heterogeneous mixture of several isoforms possibly with distinct biological activities. The main problem of using such lectins as biomedical tools is that "batch-to-batch" variation in isoforms content may lead to inconstant results. The production of lectins by recombinant means has the advantage of obtaining high amounts of proteins with defined amino-acid sequences and more precise properties. In this mini review, we provide the strategies followed to produce two different forms of frutalin in two different microbial systems: Escherichia coli and Pichia pastoris. The processing and functional properties of the recombinant frutalin obtained from these hosts are compared to those of frutalin extracted from breadfruit. Emphasis is given particularly to recombinant frutalin produced in $P$. pastoris, which showed a remarkable capacity as biomarker of human prostate cancer and as apoptosis-inducer of cancer cells. Recombinant frutalin production opens perspectives for its development as a new tool in human medicine.

Keywords: recombinant frutalin, lectin isoforms, Pichia pastoris expression system, glycosylation, Escherichia coli expression system, biomedical application, tumor biomarker, apoptosis-inducer

\section{OUTLINE}

Plant lectins have attracted much attention for biomedical applications, especially due to their remarkable anti-tumor properties, resulting from their ability to induce programmed cell death and/or autophagocytosis in cancer cells (Liu et al., 2010; Fu et al., 2011). Plant lectins are also relevant for biomedical diagnosis (Mislovicova et al., 2009).

Frutalin is a plant lectin with reported immunomodulatory (Brando-Lima et al., 2005, 2006), anti-tumor (Oliveira et al., 2011), and tumor biomarker (Oliveira et al., 2009b) properties, among other capacities (de Vasconcellos Abdon etal., 2012), and is a good example of how recombinant production of plant lectins can be challenging but also advantageous for obtaining bioactive derivatives for biomedical application. Frutalin is found in extracts of Artocarpus incisa (breadfruit) seeds (Pineau et al., 1990), from which it can be purified by affinity chromatography on cross-linked Adenanthera pavonina galactomannan (Moreira et al., 1998). The name "frutalin" (hereinafter referred to as FTL) is a composite of part of the Portuguese common name of the lectin source plant ("fruta" of "fruta-pão") followed by the suffix "-lin". Although having sugar-binding preference toward D-galactose, FTL presents a rather broad sugar-binding activity, interacting also with other sugars, as D-mannose and D-glucose. FTL belongs to the jacalin-related lectins family (JRLs, found in the Moraceae plant family), specifically to the sub-family of the galactose-specific lectins (gJRLs), as it presents high structural homology, sugar specificity and sequential identity with jacalin (the galactose-specific lectin of Artocarpus integrifolia seeds - jackfruit, the first member of this family to be identified; Pineau et al.,
1990; Moreira et al., 1998; Oliveira et al., 2009a). FTL is characterized by a strong and identical agglutinating activity with human erythrocytes of the ABO system and rabbit erythrocytes, which has no requirements for divalent metal cations (Moreira et al., 1998). Interestingly, the hemagglutination activity (HA) of FTL is three times higher than that of jacalin (Nobre et al., 2010). FTL has a sophisticated processing. The conversion of the primary translation product of gJRL-mRNA into the protein includes a complex series of co- and post-translational modifications including the removal of the signal peptide (vacuolar targeting), a (partial) glycosylation, removal of the $N$-terminal propeptide, the excision of a linker tetrapeptide, to separate two polypeptide chains ( $\alpha$ and $\beta$ ), proper folding and oligomer assembly. Molecular cloning of the FTL cDNA (excluding signal and propeptide), revealed that, as jacalin, it may be encoded by a family of genes, each of them containing $471 \mathrm{bp}$, corresponding to a protein of 157 aminoacids, with a calculated molecular weight of $17.1 \mathrm{kDa}$ (Oliveira et al., 2009a). Twenty amino-acids correspond to the $\beta$-chain, 4 amino-acids to the linker "T-S-S-N" and 133 amino-acids correspond to the $\alpha$-chain (from $N$ - to $C$-terminal). Several gJRLs conserved regions of amino-acids were found in FTL sequences, including the linker. The linker, and its processing, is specific for the sub-group of the gJRLs, being absent in the other subgroup (mannose-specific JRLs; Houles Astoul et al., 2002). FTL is a heterogeneous mixture of several slightly different amino-acid sequences sharing 93-97\% of identity, with or without consensus sequences for $N$-glycosylation (Asn-X-Thr/Ser) in the $\alpha$-chain (one of these potential $N$-glycosylation sites was also reported for jacalin; Oliveira et al., 2009a). In fact, FTL is a partly glycosylated 
protein, with $2.1 \%$ of carbohydrates (Moreira et al., 1998). Different FTL isoforms or iso-lectins (i.e., different mature sequences) may have distinct biological activities, as reported for other plant lectins (Raemaekers et al., 1999; Ohba et al., 2003). Under denaturing conditions (SDS-PAGE), FTL presents two bands: the upper band $(15.5 \mathrm{kDa})$ corresponds to the highly glycosylated isoforms of $\alpha$ chain, whereas the lower band $(12 \mathrm{kDa})$ represents the slightly or non-glycosylated isoforms of the same chain (Oliveira et al., 2008). The $\beta$ chain is not visible due to its low molecular weight (2.1 kDa; Oliveira et al., 2008). In its native form, FTL is a tetrameric molecule, consisting of four monomers bound by noncovalent linkages, each containing one $\beta$ and one $\alpha$ chain, forming four sugar-binding sites, with a predominantly $\beta$ sheet conformation (Moreira et al., 1998; Campana et al., 2002) and an apparent molecular mass of 48-49 kDa (Moreira et al., 1998; Oliveira et al., 2008). FTL is a robust protein as it is stable up to $60^{\circ} \mathrm{C}$ and very resistant to chemical denaturation (Campana et al., 2002).

Plant lectins are commonly isolated from their natural sources, although this presents several disadvantages, as the resulting isoforms. Recombinant production, mainly in microbial hosts, is an interesting way to overcome this problem, whilst it may allow to improve availability, ensure continuous supply and facilitate purification of lectins with interesting activities or improved/tailor-made functionalities, particularly for biomedical application (for a recent review see Oliveira et al., 2013).

This review describes the case study of the different strategies applied for the production of FTL in the bacterium Escherichia coli (Oliveira et al., 2009a; Costa, 2013; Costa et al., 2013a) and in the yeast Pichia pastoris (Oliveira et al., 2008). Several variables were considered for optimization: codon usage, strains, fusion partners, induction conditions, and purification methodology. Both microorganisms are well-established platforms for the production of recombinant proteins, including several approved biopharmaceutical products (Berlec and Strukelj, 2013; Gasser et al., 2013). These are also the most employed hosts for the production of recombinant lectins, namely plant lectins for biomedical purposes, such as jacalin (Sahasrabuddhe etal., 2004, 2006), aviscumin (from Viscum album; Zwierzina et al., 2011), PCL (from Polygonatum cyrtonema; Li et al., 2011), Orysata (from Oryza sativa; Al Atalah et al., 2011), and GNA maize (from Galanthus nivalis; Fouquaert etal., 2009). E. coli is commonly used to produce non-glycosylated lectins, while $P$. pastoris is mainly employed to overcome problems of insoluble expression of the bacterial system and to produce glycosylated lectins. Thus, E. coli and P. pastoris were chosen to produce non-glycosylated recombinant frutalin (EcrFTL) and glycosylated recombinant frutalin (PprFTL), respectively. The bio-molecular characterization of the recombinant FTL obtained from each host in terms of processing, molecular weight, HA and sugar-binding activity is herein presented. Finally, a main focus is given to PprFTL due to its demonstrated anti-tumor and tumor biomarker activities (Oliveira et al., 2009b, 2011).

\section{PRODUCTION OF RECOMBINANT FRUTALIN IN E. coli}

A FTL cDNA sequence was used for production of recombinant FTL in E. coli by different strategies (Oliveira et al., 2009a). The first attempts to produce soluble EcrFTL in E. coli focused in the use of engineered E. coli strains that have extra copies of rare
tRNAs and in the optimization of the induction conditions, but resulted in low yields (Oliveira et al., 2009a; Costa, 2013). The soluble production of EcrFTL from strain E. coli BL21 Codon Plus RIPL (DE3), harboring the pET-25b $(+)$ expression vector (Novagen), was maximized to $16 \mathrm{mg} / \mathrm{l}$ by the implementation of an experimental factorial design (Oliveira et al., 2009a; Figure 1). However, all the experimental conditions resulted in EcrFTL produced predominantly as insoluble protein. Even though, EcrFTL was purified from crude $E$. coli extracts by sequential size exclusion (SEC) and cation ion exchange chromatography (IEC) that yielded $76 \mu \mathrm{g}$ of protein per liter of E. coli culture. Purified EcrFTL migrated in SDS-PAGE gel as a homogeneous single-band protein with a molecular mass of about $17 \mathrm{kDa}$, indicating that the linker was not cleaved. Nevertheless, EcrFTL presented HA against rabbit erythrocytes, although it required more time to develop this activity than FTL. Thus, the HA of FTL is not strictly dependent on linker cleavage. In assays of HA inhibition by different sugars, EcrFTL presented specificity for galactose; however, it could not be purified by affinity chromatography on A. pavonina galactomannan, thus revealing lower sugar-binding affinity than FTL. The biomedical properties of this EcrFTL were not evaluated since the amounts obtained through this strategy were unsatisfactory and we were willing to improve them.

Taking into account the low production yields previously obtained, fusion protein technology was afterward considered to improve the soluble production and purification of recombinant FTL in E. coli. Eight fusion tags ( $\mathrm{His}_{6}$, Trx, GST, NusA, MBP, SUMO, $\mathrm{H}$, and Fh8), included in pETM vectors (EMBL), were evaluated in small-scale screening assays for recombinant FTL solubility in four E. coli strains (Costa et al., 2013a; Figure 1). All vectors provided a $\mathrm{His}_{6}$ tag for purification of the EcrFTL fusions by nickel affinity (immobilized metal ion affinity chromatography - IMAC). The Rosetta strain (DE3) was selected for scale-up protein processing, namely purification and solubility evaluation before and after tag cleavage. The solubility enhancer partners NusA, Trx, and Fh8 tags considerably improved the soluble production of EcrFTL (in the order: NusA $\sim$ Fh8 $>\operatorname{Trx}$ ), being the protein soluble after their removal by TEV cleavage (Tobacco Etch Virus protease). Interestingly, the cleaved and purified EcrFTL from the Fh8 and Trx fusions presented higher amounts than that cleaved from the NusA fusion protein (Costa et al., 2013a). The fusion protein strategy boosted the availability of EcrFTL by increasing its yield from $\mu \mathrm{g}$ to $\mathrm{mg}$ of active protein per liter of E. coli culture whilst simplifying the complete production and purification protocol (Costa, 2013). IMAC revealed to be a simpler, easier and quicker procedure than SEC or IEC for EcrFTL purification, and it also decreased EcrFTL losses during purification. Moreover, EcrFTL kept its HA when fused to this partner (our unpublished results). However, the His $_{6}$ FTL fusion (produced from vector pETM-11) was found to be a very unstable protein, precipitating at physiological $\mathrm{pH}$, and thus being incompatible with cell culture conditions for evaluation of its anti-tumor activity (our unpublished results). Among the fusion partners tested, the Fh8 tag was pointed as a good option for the production of soluble EcrFTL in E. coli because of its advantageous low molecular weight and combined solubility enhancer and purification handle activities (Costa, 2013). The Fh8 


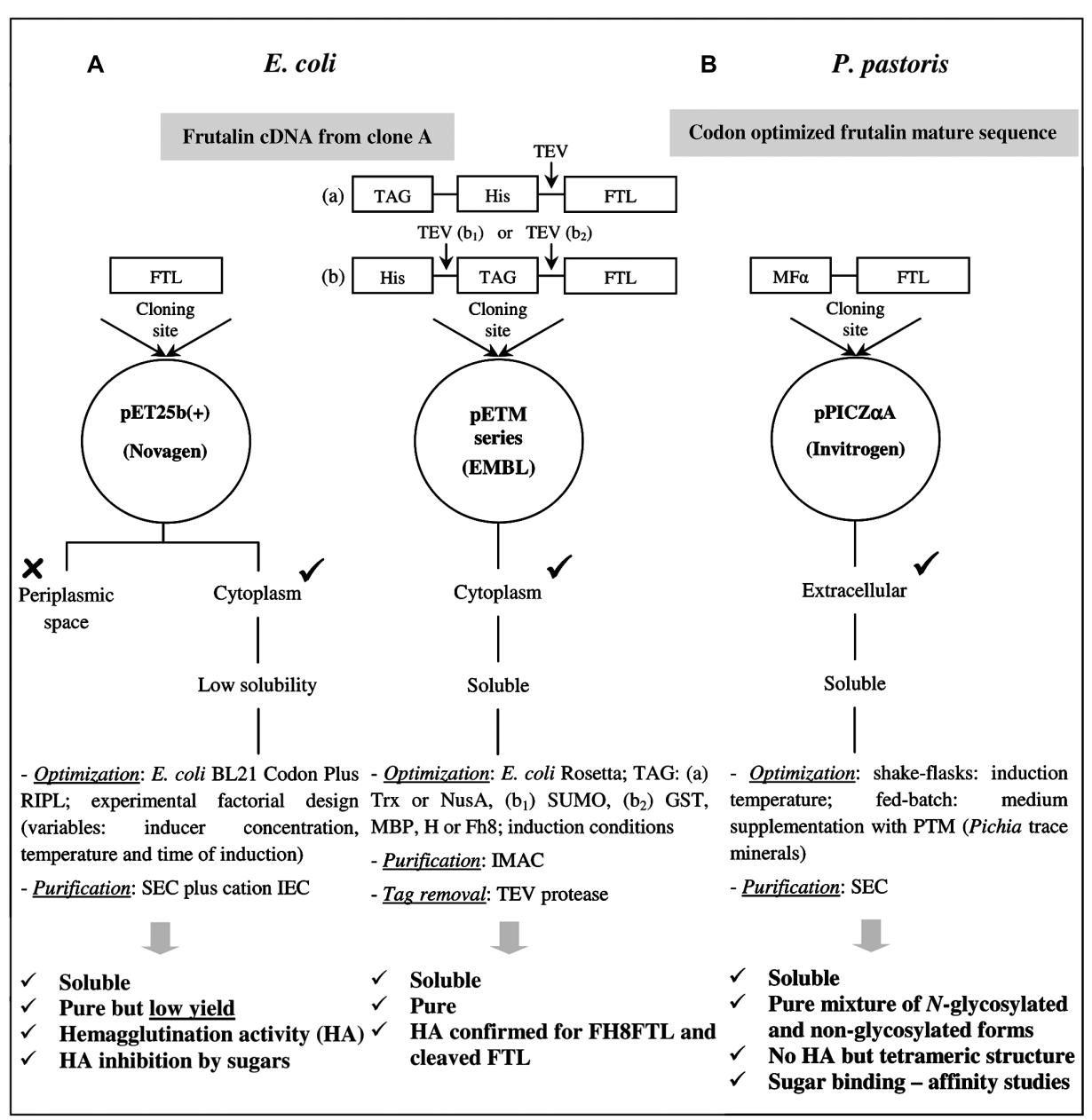

FIGURE 1 | Main strategies for the production of soluble recombinant FTL in Escherichia coli (A) and Pichia pastoris (B). Different frutalin codifying genes were cloned in E. coli and P. pastoris, which deduced amino-acid sequences share $93 \%$ of identity (Table 2 in Oliveira et al., 2009a).

tag (Fasciola hepatica 8-kDa antigen) has been recently ranked among the best solubility enhancer partners for recombinant protein production in E. coli (Costa et al., 2013a, 2014). Fh8 was also shown as a suitable fusion partner for purification of recombinant proteins in E. coli by HIC (hydrophobic interaction chromatography), with efficiencies comparable to those of IMAC (Costa et al., 2013b). Besides improving EcrFTL solubility, the Fh8 tag increased EcrFTL stability, compared to the $\mathrm{His}_{6}$ tag, and it did not interfere with the HA and structure (in respect to $\beta$-sheet conformation) of EcrFTL, before and after its cleavage (Costa, 2013). The anti-tumor activity of these two versions of EcrFTL, the Fh8FTL and Fh8-cleaved FTL, is under evaluation.

\section{PRODUCTION OF RECOMBINANT FRUTALIN IN P. pastoris PRODUCTION AND BIO-MOLECULAR CHARACTERIZATION}

Frutalin has highly glycosylated isoforms and the presence of the glycans may be important for its functional properties. Thus, we planned to produce FTL in a microorganism capable of performing glycosylation, namely using the strain $P$. pastoris
KM71H (Oliveira et al., 2008; Figure 1). FTL gene (encoding a mature sequence; Oliveira et al., 2009a) was optimized based on the codon usage of $P$. pastoris and integrated into the yeast genome in frame at the $C$-terminal of the Saccharomyces $\alpha$-factor preprosequence $(M F \alpha)$, to direct PprFTL into the secretory pathway, and under the control of the strong methanol inducible $A O X 1$ promoter. PprFTL was produced in shake-flasks and purified from supernatants by SEC yielding 18-20 mg per liter of culture.

Important differences between the molecular and biological properties of PprFTL and FTL were found (Oliveira et al., 2008). The reason for that was the processing of FTL in P. pastoris, which was different from that occurring in breadfruit. As also observed in E. coli (Oliveira et al., 2009a), the FTL linker was not cleaved in P. pastoris (confirmed by $\mathrm{N}$-terminal sequencing), thus suggesting that this processing can be specific for higher eukaryotes. Furthermore, the MF $\alpha$ secretion leader was incompletely removed, resulting in PprFTL with one Glu-Ala repeat at its $N$-terminal, decreasing its predicted PI (isoeletric point) from 8 to 5 . These repeats are commonly observed in heterologous proteins secreted by P. pastoris using this signal sequence, and also reported for other 
recombinant plant lectins (Raemaekers et al., 1999; Lannoo et al., 2007).

As expected, PprFTL was $N$-glycosylated by $P$. pastoris, since the corresponding protein sequence has one potential site for $\mathrm{N}$ glycosylation ( $\alpha$-Asn74). Part of the secreted PprFTL undergone this post-translational modification, which led to an extension in its molecular weight of about $2.8 \mathrm{kDa}$ (Oliveira et al., 2011). PprFTL, contrarily to FTL, did not agglutinate rabbit erythrocytes, despite also having a tetrameric structure (Oliveira et al., 2008). Thus, it was hypothesized that glycosylation pattern of $P$. pastoris inhibited this activity since non-glycosylated EcrFTL presented HA. In a previous work, Pichia glycosylation was also suggested to inhibit the HA of a fungal lectin (Iijima et al., 2003). Nevertheless, it should be noted that different FTL coding sequences were cloned in P. pastoris and E. coli, and hence the HA of the resulting proteins may differ. The deduced amino-acid sequences of the frutalin codifying genes cloned in $P$. pastoris and E. coli have $93 \%$ of sequence identity (Figure 1). In what concerns carbohydrate-binding activity, PprFTL presented a sugar preference similar to FTL, but with less affinity. The affinity constant for the binding of PprFTL to the monosaccharide methyl- $\alpha$-galactose was determined and found to be 113-fold lower than that of FTL (Oliveira et al., 2008). The only other gJRL so far produced in microorganisms was jacalin. Jacalin was produced in E. coli also as an unprocessed protein with its sugar-binding activities reduced in the same order of magnitude (Sahasrabuddhe et al., 2004). The correct excision of the linker and consequent generation of a free glycine at the $N$-terminus of the $\alpha$ chain may determine gJRLs sugar-binding properties (Houles Astoul et al., 2002; Sahasrabuddhe et al., 2004; Oliveira et al., 2008).

The large-scale production of PprFTL was conducted in a 1.6 $\mathrm{L}$ stirred tank bioreactor operating in fed-batch mode at $28^{\circ} \mathrm{C}$ during 4 days (Wanderley et al., 2013). Supplementation of the culture medium (BMMH - buffered minimal methanol medium) with Pichia trace minerals (PTM) resulted in 2.5-fold higher PprFTL production $(13.4 \mathrm{mg} / \mathrm{l})$ than that achieved without supplementation $(5.23 \mathrm{mg} / \mathrm{l})$. Furthermore, bioreactor resulted in fourfold higher PprFTL production, comparing to shaker-flasks batch assays $(3.3 \mathrm{mg} / \mathrm{l})$, using the same culture medium (BMMH plus PTM) and induction conditions (Wanderley et al., 2013). Nevertheless, the yield of PprFTL was higher from shake-flasks induced at $15^{\circ} \mathrm{C}(18-20 \mathrm{mg} / \mathrm{l})$, which means that lower temperatures favor PprFTL production (Oliveira et al., 2008). However, 20 of such flasks (each containing $50 \mathrm{ml}$ of BMMH medium) are needed to obtain the same amount of PprFTL as in one fed-batch experiment. Thus, bioreactor fermentation is more advantageous for the production of PprFTL.

\section{BIOMEDICAL PROPERTIES}

The relevance of JRLs, specifically jacalin, for cancer diagnostics and therapeutics is present in many recent works (e.g., Obaid et al., 2012; Lee et al., 2013; Marangoni et al., 2013; Zupancic et al., 2014). PprFTL was evaluated in terms of its tumor biomarker and antitumor properties, comparatively to FTL (Oliveira et al., 2009b, 2011). The cancer biomarker study was performed by immunohistochemistry with human prostate tissues (Oliveira et al., 2009b). Other plant lectins were used in the past in similar studies but with limited success (works cited in Oliveira et al., 2009b). The binding pattern of PprFTL and FTL to the prostate tissues was distinct, presumably due to their differences in carbohydrate-binding affinity (Oliveira et al., 2008). FTL bound to any type of prostate cells but more strongly to the neoplasic (malignant cells) than to the hyperplasic ones (non-malignant cells). On the other hand, PprFTL was much more specific, as it just recognized malignant cells (Figure 2). A significant positive statistical correlation between the binding intensity of PprFTL and the histological diagnosis of the tissues was obtained (not observed for FTL), although PprFTL did not recognize all the malignant cases studied (30\% had negative binding), and when positive, the binding was heterogeneous. However, only a small number of prostate cases were analyzed and the histochemical methodology has still room for improvement. This study indicates that PprFTL has higher potential as cancer biomarker than FTL.

In in vitro assays, PprFTL showed a strong cytotoxic effect on HeLa cervical cancer cells proliferation, by inducing cell dead by apoptosis (Oliveira et al., 2011). This effect was irreversible as well as time and dose dependent $\left(\mathrm{IC}_{50} \sim 100 \mu \mathrm{g} / \mathrm{mL}\right)$. Identical results were obtained for FTL in the same study (Oliveira et al., 2011).

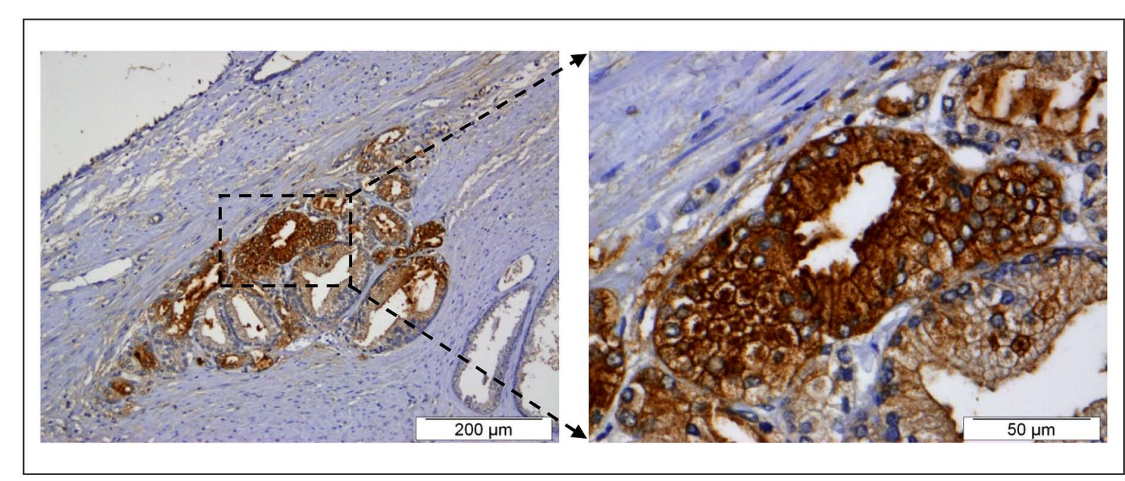

FIGURE 2 | Immunostaining pictures of a prostate cancer tissue using recombinant FTL produced in $\boldsymbol{P}$ pastoris as tumor diagnostic marker. PprFTL was able to specifically recognize carcinoma cells in middle of a benign lesion. The staining is localized in the cells cytoplasm of the carcinoma glands (brown color). (Original publisher: BioMed Central; Adapted from Oliveira et al., 2009b). 
Thus, it seems that frutalin action in tumor cells is not exclusively dependent on its carbohydrate-binding properties. Other factors, such as protein-protein interactions, may contribute to the cellular responses, as suggested for the effect of recombinant jacalin in tumor cells (Sahasrabuddhe et al., 2006). Recombinant jacalin showed a magnitude of anti-proliferative responses similar to native jacalin on human cancer cell lines, despite its inferior sugar-binding affinity (Sahasrabuddhe et al., 2006). Both, PprFTL and FTL showed nuclear migration activity on HeLa cells (Oliveira et al., 2011), a property only reported for fungal lectins (Yu et al., 1999; Francis et al., 2003; Liang et al., 2009). Plant lectins have been described to attach to cancer cells membrane [e.g., jacalin in A431 human epidermoid carcinoma cells (Sahasrabuddhe et al., 2006)] or to be internalized and located in different cellular compartments [e.g., wheat germ agglutinin, WGA, in DU-145 human prostate cancer cells (Gabor et al., 2001)]. To our knowledge, our work is the first reporting nuclear migration activity on cancer cells for a lectin from plant origin (Oliveira et al., 2011). Studies aiming to elucidate the apoptotic mechanism triggered by PprFTL on cancer cells are now being conducted.

\section{CONCLUSION AND PROSPECTS}

Escherichia coli and Pichia pastoris were found as suitable hosts for producing high amounts of recombinant FTL upon production and purification optimization. Optimization in E. coli significantly improved EcrFTL production, leading to high yields, but decreased protein stability. Furthermore, the processing of recombinant FTL in both microorganisms was different from that occurring in breadfruit, resulting in versions of FTL with inferior HA and carbohydrate-binding capacity. Nevertheless, PprFTL presented an anti-tumor activity identical to FTL and enhanced tumor biomarker capacity. The production strategies herein presented will extend the research on the biomedical properties of recombinant FTL.

The importance of amino-acids substitutions and posttranslational modifications in gJRLs (e.g., linker cleavage, glycosylation) remains to be elucidated, and thus, future research might follow this direction. Within this scope, production and the availability of heterologous recombinant lectins is a valuable tool that can contribute to the fundamental understanding of the biological activity of the lectins. The production of the same lectin coding sequence in prokaryotic and eukaryotic hosts, the production of different lectin isoforms, and engineered/mutated versions, will provide insight into lectins functionality and shed light into its native physiological role. Furthermore, recombinant lectins with refined properties can be obtained. Finally, the fusion of enhanced lectins with functional moieties, by using recombinant DNA technology, for the development of functionalized drug delivery systems for site specific anti-tumor therapy, is anticipated.

\section{AUTHOR CONTRIBUTIONS}

Carla Oliveira drafted the review and carried out most of the experimental work of recombinant FTL. José A. Teixeira participated in the development of the concept. Lucília Domingues conceived the study and helped to draft the review. All authors read and approved the final manuscript.

\section{ACKNOWLEDGMENTS}

Carla Oliveira acknowledges support from Fundação para a Ciência e a Tecnologia (FCT), Portugal (by the fellowship SFRH/BDP/63831/2009). The authors thank the FCT GlycoCBMs Project REF. FCT PTDC/AGR-FOR/3090/2012 FCOMP-01-0124-FEDER-027948, the FCT Strategic Project PEstOE/EQB/LA0023/2013, and the Project "BioInd - Biotechnology and Bioengineering for improved Industrial and Agro-Food processes," REF. NORTE-07-0124-FEDER-000028 Co-funded by the Programa Operacional Regional do Norte (ON.2 - O Novo Norte), QREN, FEDER.

\section{REFERENCES}

Al Atalah, B., Fouquaert, E., Vanderschaeghe, D., Proost, P., Balzarini, J., Smith, D. F., etal. (2011). Expression analysis of the nucleocytoplasmic lectin 'Orysata' from rice in Pichia pastoris. FEBS J. 278, 2064-2079. doi: 10.1111/j.1742-4658.2011.08122.x

Berlec, A., and Strukelj, B. (2013). Current state and recent advances in biopharmaceutical production in Escherichia coli, yeasts and mammalian cells. J. Ind. Microbiol. Biotechnol. 40, 257-274. doi: 10.1007/s10295-013-1235-0

Brando-Lima, A. C., Saldanha-Gama, R. F., Henriques, M., Monteiro-Moreira, A. C., Moreira, R. A., and Barja-Fidalgo, C. (2005). Frutalin, a galactose-binding lectin, induces chemotaxis and rearrangement of actin cytoskeleton in human neutrophils: involvement of tyrosine kinase and phosphoinositide 3-kinase. Toxicol. Appl. Pharmacol. 208, 145-154. doi: 10.1016/j.taap.2005.02.012

Brando-Lima, A. C., Saldanha-Gama, R. F., Pereira, C. R., Villela, C. G., Sampaio, A. L., Monteiro-Moreira, A. C., et al. (2006). Involvement of phosphatidylinositol-3 kinase-Akt and nuclear factor kappa-B pathways in the effect of frutalin on human lymphocyte. Int. Immunopharmacol. 6, 465-472. doi: 10.1016/j.intimp.2005.09.008

Campana, P. T., Moraes, D. I., Monteiro-Moreira, A. C., and Beltramini, L. M. (2002). Unfolding and refolding studies of frutalin, a tetrameric D-galactose binding lectin. Eur. J. Biochem. 269, 753-758. doi: 10.1046/j.0014-2956.2002.02742.x

Costa, S. (2013). Development of A Novel Fusion System for Recombinant Protein Production and Purification In Escherichia coli. Ph.D. thesis, University of Minho, Braga.

Costa, S., Almeida, A., Castro, A., and Domingues, L. (2014). Fusion tags for protein solubility, purification and immunogenicity in Escherichia coli: the novel Fh8 system. Front. Microbiol. 5:63. doi: 10.3389/fmicb.2014.00063

Costa, S. J., Almeida, A., Castro, A., Domingues, L., and Besir, H. (2013a). The novel Fh8 and $\mathrm{H}$ fusion partners for soluble protein expression in Escherichia coli: a comparison with the traditional gene fusion technology. Appl. Microbiol. Biotechnol. 97, 6779-6791. doi: 10.1007/s00253-012-4559-1

Costa, S. J., Coelho, E., Franco, L., Almeida, A., Castro, A., and Domingues, L. (2013b). The Fh8 tag: a fusion partner for simple and cost-effective protein purification in Escherichia coli. Protein Expr. Purif. 92, 163-170. doi: 10.1016/j.pep.2013.09.013

de Vasconcellos Abdon, A. P., Coelho De Souza, G., Noronha Coelho De Souza, L., Prado Vasconcelos, R., Araujo Castro, C., Moreira Guedes, M., etal. (2012). Gastroprotective potential of frutalin, a D-galactose binding lectin, against ethanol-induced gastric lesions. Fitoterapia 83, 604-608. doi: 10.1016/j.fitote.2012.01.005

Fouquaert, E., Smith, D. F., Peumans, W. J., Proost, P., Balzarini, J., Savvides, S. N., et al. (2009). Related lectins from snowdrop and maize differ in their carbohydrate-binding specificity. Biochem. Biophys. Res. Commun. 380, 260-265. doi: 10.1016/j.bbrc.2009.01.048

Francis, F., Marty-Detraves, C., Poincloux, R., Baricault, L., Fournier, D., and Paquereau, L. (2003). Fungal lectin, XCL, is internalized via clathrin-dependent endocytosis and facilitates uptake of other molecules. Eur. J. Cell Biol. 82, 515-522. doi: 10.1078/0171-9335-8

Fu, L. L., Zhou, C. C., Yao, S., Yu, J. Y., Liu, B., and Bao, J. K. (2011). Plant lectins: targeting programmed cell death pathways as antitumor agents. Int. J. Biochem. Cell Biol. 43, 1442-1449. doi: 10.1016/j.biocel.2011.07.004

Gabor, F., Klausegger, U., and Wirth, M. (2001). The interaction between wheat germ agglutinin and other plant lectins with prostate cancer cells Du-145. Int. J. Pharm. 221, 35-47. doi: 10.1016/S0378-5173(01)00650-0 
Gasser, B., Prielhofer, R., Marx, H., Maurer, M., Nocon, J., Steiger, M., et al. (2013). Pichia pastoris: protein production host and model organism for biomedical research. Future Microbiol. 8, 191-208. doi: 10.2217/fmb.12.133

Houles Astoul, C., Peumans, W. J., Van Damme, E. J., Barre, A., Bourne, Y., and Rouge, P. (2002). The size, shape and specificity of the sugar-binding site of the jacalin-related lectins is profoundly affected by the proteolytic cleavage of the subunits. Biochem. J. 367(Pt 3), 817-824. doi: 10.1042/BJ20020856

Iijima, N., Amano, K., Ando, A., and Nagata, Y. (2003). Production of fruiting-body lectins of Pleurotus cornucopiae in methylotrophic yeast Pichia pastoris. J. Biosci. Bioeng. 95, 416-418. doi: 10.1016/S1389-1723(03)80079-8

Lannoo, N., Vervecken, W., Proost, P., Rouge, P., and Van Damme, E. J. M. (2007). Expression of the nucleocytoplasmic tobacco lectin in the yeast Pichia pastoris. Protein Expr. Purif. 53, 275-282. doi: 10.1016/j.pep.2007.01.007

Lee, C. S., Muthusamy, A., Abdul-Rahman, P. S., Bhavanandan, V. P., and Hashim, O. H. (2013). An improved lectin-based method for the detection of mucin-type Oglycans in biological samples. Analyst 138, 3522-3529. doi: 10.1039/c3an36258b

Li, C. Y., Luo, P., Liu, J. J., Wang, E. Q., Li, W. W., Ding, Z. H., et al. (2011). Recombinant expression of Polygonatum cyrtonema lectin with anti-viral, apoptosis-inducing activities and preliminary crystallization. Process Biochem. 46, 533-542. doi: 10.1016/j.procbio.2010.10.005

Liang, Y., Feng, L., Tong, X., Wang, K., Li, D. F., Lin, J. C., et al. (2009). Importance of nuclear localization for the apoptosis-induced activity of a fungal galectin AAL (Agrocybe aegerita lectin). Biochem. Biophys. Res. Commun. 386, 437-442. doi: 10.1016/j.bbrc.2009.06.054

Liu, B., Bian, H. J., and Bao, J. K. (2010). Plant lectins: potential antineoplastic drugs from bench to clinic. Cancer Lett. 287, 1-12. doi: 10.1016/j.canlet.2009.05.013

Marangoni, V. S., Paino, I. M., and Zucolotto, V. (2013). Synthesis and characterization of jacalin-gold nanoparticles conjugates as specific markers for cancer cells. Colloids Surf. B Biointerfaces 112, 380-386. doi: 10.1016/j.colsurfb.2013. 07.070

Mislovicova, D., Gemeiner, P., Kozarova, A., and Kozar, T. (2009). Lectinomics I. Relevance of exogenous plant lectins in biomedical diagnostics. Biologia 64, 1-19. doi: 10.2478/s11756-009-0029-3

Moreira, R. A., Castelo-Branco, C. C., Monteiro, A. C., Tavares, R. O., and Beltramini, L. M. (1998). Isolation and partial characterization of a lectin from Artocarpus incisa L. seeds. Phytochemistry 47, 1183-1188. doi: 10.1016/S00319422(97)00753-X

Nobre, T. M., Pavinatto, F. J., Cominetti, M. R., Selistre De-Araujo, H. S., Zaniquelli, M. E., and Beltramini, L. M. (2010). The specificity of frutalin lectin using biomembrane models. Biochim. Biophys. Acta 1798, 1547-1555. doi: 10.1016/j.bbamem.2010.03.021

Obaid, G., Chambrier, I., Cook, M. J., and Russell, D. A. (2012). Targeting the oncofetal Thomsen-Friedenreich disaccharide using jacalin-PEG phthalocyanine gold nanoparticles for photodynamic cancer therapy. Angew. Chem. Int. Ed. Engl. 51, 6158-6162. doi: 10.1002/anie.201201468

Ohba, H., Bakalova, R., and Muraki, M. (2003). Cytoagglutination and cytotoxicity of Wheat Germ Agglutinin isolectins against normal lymphocytes and cultured leukemic cell lines - relationship between structure and biological activity. Biochim. Biophys. Acta 1619, 144-150. doi: 10.1016/S0304-4165(02) 00479-8

Oliveira, C., Costa, S., Teixeira, J. A., and Domingues, L. (2009a). cDNA cloning and functional expression of the $\alpha$-D-galactose-binding lectin frutalin in Escherichia coli. Mol. Biotechnol. 43, 212-220. doi: 10.1007/s12033-0099191-7

Oliveira, C., Felix, W., Moreira, R. A., Teixeira, J. A., and Domingues, L. (2008). Expression of frutalin, an $\alpha$-D-galactose-binding jacalin-related lectin, in the yeast Pichia pastoris. Protein Expr. Purif. 60, 188-193. doi: 10.1016/j.pep.2008. 04.008
Oliveira, C., Nicolau, A., Teixeira, J. A., and Domingues, L. (2011). Cytotoxic effects of native and recombinant frutalin, a plant galactose-binding lectin, on HeLa cervical cancer cells. J. Biomed. Biotechnol. 2011, 568932. doi: $10.1155 / 2011 / 568932$

Oliveira, C., Teixeira, J. A., and Domingues, L. (2013). Recombinant lectins: an array of tailor-made glycan-interaction biosynthetic tools. Crit. Rev. Biotechnol. 33, 66-80. doi: 10.3109/07388551.2012.670614

Oliveira, C., Teixeira, J. A., Schmitt, F., and Domingues, L. (2009b). A comparative study of recombinant and native frutalin binding to human prostate tissues. $B M C$ Biotechnol. 9:78. doi: 10.1186/1472-6750-9-78

Pineau, N., Pousset, J. L., Preud'homme, J. L., and Aucouturier, P. (1990). Structural and functional similarities of breadfruit seed lectin and jacalin. Mol. Immunol. 27, 237-240. doi: 10.1016/0161-5890(90)90135-M

Raemaekers, R. J. M., De Muro, L., Gatehouse, J. A., and Fordham-Skelton, A. P. (1999). Functional phytohemagglutinin (PHA) and Galanthus nivalis agglutinin (GNA) expressed in Pichia pastoris - Correct N-terminal processing and secretion of heterologous proteins expressed using the PHA-E signal peptide. Eur. J. Biochem. 265, 394-403. doi: 10.1046/j.1432-1327.1999.00749.x

Sahasrabuddhe, A. A., Ahmed, N., and Krishnasastry, M. V. (2006). Stress-induced phosphorylation of caveolin-1 and p38, and down-regulation of EGFr and ERK by the dietary lectin jacalin in two human carcinoma cell lines. Cell Stress Chaperones 11, 135-147. doi: 10.1379/CSC-160R.1

Sahasrabuddhe, A. A., Gaikwad, S. M., Krishnasastry, M. V., and Khan, M. I. (2004). Studies on recombinant single chain jacalin lectin reveal reduced affinity for saccharides despite normal folding like native jacalin. Protein Sci. 13, 3264-3273. doi: 10.1110/ps.04968804

Wanderley, M. S. O., Oliveira, C., Bruneska, D., Domingues, L., Lima, J. L., Teixeira, J. A., et al. (2013). Influence of trace elements supplementation on the production of recombinant frutalin by Pichia pastoris KM71H in fed-batch process. Chem. Pap. 67, 682-687. doi: 10.2478/s11696-013-0363-3

Yu, L. G., Fernig, D. G., White, M. R. H., Spiller, D. G., Appleton, P., Evans, R. C., etal. (1999). Edible mushroom (Agaricus bisporus) lectin, which reversibly inhibits epithelial cell proliferation, blocks nuclear localization sequence-dependent nuclear protein import. J. Biol. Chem. 274, 4890-4899. doi: 10.1074/jbc.274.8.4890

Zupancic, D., Kreft, M. E., and Romih, R. (2014). Selective binding of lectins to normal and neoplastic urothelium in rat and mouse bladder carcinogenesis models. Protoplasma 251, 49-59. doi: 10.1007/s00709-013-0524-9

Zwierzina, H., Bergmann, L., Fiebig, H., Aamdal, S., Schoffski, P., Witthohn, K., et al. (2011). The preclinical and clinical activity of aviscumine: a potential anticancer drug. Eur. J. Cancer 47, 1450-1457. doi: 10.1016/j.ejca.2011.02.022

Conflict of Interest Statement: The authors declare that the research was conducted in the absence of any commercial or financial relationships that could be construed as a potential conflict of interest.

Received: 31 May 2014; accepted: 22 July 2014; published online: 08 August 2014. Citation: Oliveira C, Teixeira JA and Domingues L (2014) Recombinant production of plant lectins in microbial systems for biomedical application - the frutalin case study. Front. Plant Sci. 5:390. doi: 10.3389/fpls.2014.00390

This article was submitted to Plant Physiology, a section of the journal Frontiers in Plant Science.

Copyright (c) 2014 Oliveira, Teixeira and Domingues. This is an open-access article distributed under the terms of the Creative Commons Attribution License (CC BY). The use, distribution or reproduction in other forums is permitted, provided the original author(s) or licensor are credited and that the original publication in this journal is cited, in accordance with accepted academic practice. No use, distribution or reproduction is permitted which does not comply with these terms. 